\title{
REVISIÓN DE SEIS INSCRIPCIONES SOBRE CERÁMICA PROCEDENTES DE AZAILA (TERUEL) ${ }^{1}$
}

\author{
Juana Valladolid
}

1. Hace unos años tuve la oportunidad de revisar los materiales epigráficos procedentes del yacimiento del Cabezo de Alcalá de Azaila (Teruel) que custodia el Museo Arqueológico Nacional (MAN) ${ }^{2}$ y que han sido objeto de estudio con mayor o menor extensión y profundidad en diversas publicaciones ${ }^{3}$ hasta su completa corporización en el volumen III de los Monumenta Linguarum Hispanicarum (=MLH) de J. Untermann ${ }^{4}$. En la actualidad, y en el marco del Proyecto de Banco de Datos Lingüísticos Paleohispánicos ${ }^{5}$, volvemos a retomar su análisis.

Los calcos obtenidos ofrecen transcripciones idénticas a las ya conocidas a través de los $M L H$, salvo en un pequeño número de inscripciones cuya lectura difiere en alguno de los signos. En la mayoría de los casos la nueva transcripción no modifica el sentido del epígrafe (en su mayoría probables marcas de propiedad de contenido léxico inservible); sin embargo, hay 6 que creemos conveniente comentar, algunos por sus peculiaridades paleográficas y otros por tratarse de secuencias más largas que las habituales en Azaila y por tanto ser susceptibles de encerrar algún tipo de información no extraible de los grafitos cortos.

2. Iniciamos ya el repaso de los epígrafes que se presentarán con una entrada que incluye la referencia de $\operatorname{los} M L H$ y su transcripción en ese corpus, la nueva lectura, el número con el que está inventariado en el MAN y el envío a la figura correspondiente. Se describe a continuación el soporte ampliando los datos recogidos en $M L H$ y se aborda el comen-

\footnotetext{
${ }^{1}$ Este trabajo se realiza dentro del proyecto Hesperia BFF2000-0692-C02-01

${ }^{2}$ Agradezco al $\mathrm{Dpt}^{\mathrm{o}}$ de Protohistoria del MAN las facilidades que en todo momento me otorgaron para el estudio de las piezas y en definitiva para la realización de mi Memoria de Licenciatura.

${ }^{3}$ Cabré (1926, 1944); Beltrán Lloris, M. (1976, 1979).

${ }^{4}$ Con posterioridad a la fecha de publicación de MLH III (1990), se ha dado a conocer (Beltrán Lloris, M., 1995) documentación manuscrita de J. Cabré Aguiló correspondiente a sus primeras campañas de excavación en Azaila.

${ }^{5}$ Cit. nota 1
} 


\section{Juana Valladolid}

tario epigráfico prestando especial atención a la paleografía. Las oportunas referencias bibliográficas cerrarán cada uno de los comentarios.

\section{E. 1.223: ]tiko}

Nueva lectura: ]tiśker o (]tiśkeŕ)

MAN 1943/69/226

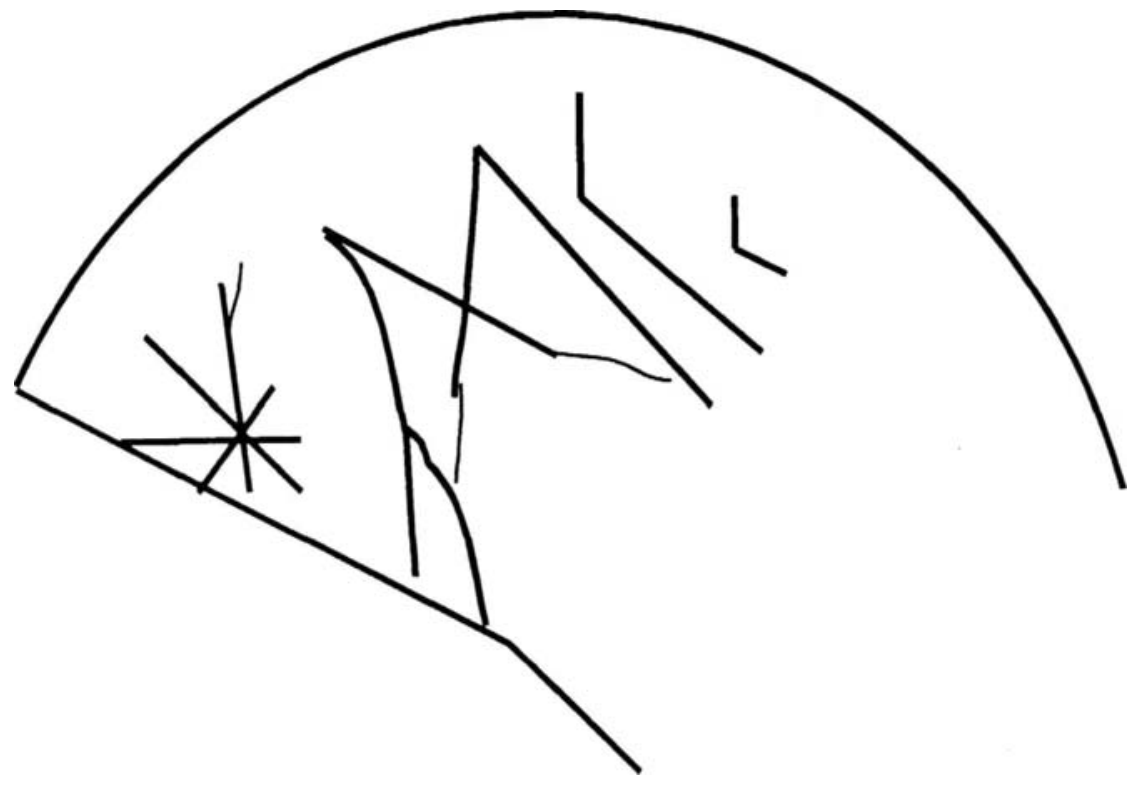

Figura l. E. 1.223

Gran fragmento de base de cerámica campaniense B, forma 1 de Lamboglia (=Lamb.), tiene un diámetro de 11,2 cm., la pasta es beige y el barniz denso en el interior y prácticamente desaparecido en el fondo exterior.

La inscripción, grabada postcocción en el fondo exterior, desde el contorno hacia el interior, consta de 3 grafemas completos y uno incompleto, dispuestos en una línea de $45 \mathrm{~mm}$ de longitud (desde la línea de fractura, que coincide con el inicio del primer signo, hasta la interrupción del último signo). Está posiblemente incompleta por la izquierda.

El primer signo, de trazos anchos y profundos, tiene una altura de $19 \mathrm{~mm}$ y se presenta bajo una forma poco frecuente - ti6- ${ }^{6}$ que en Azaila sólo se repite en otro grafito (E. 1.265). Los dos trazos oblicuos se prolongan por debajo del punto de intersección con el central y el derecho se bifurca en su extremo superior. El segundo signo, ś2, prolonga todos sus trazos más allá de lo que un dibujo ortodoxo del mismo exigiría, los oblicuos internos llegan a invadir los externos ofreciendo un aspecto final que se aproxima al del signo ko (variante ko3). Sin embargo, la

${ }^{6}$ Según MLHIII, 1, tabla 2, pp 246-247. 
incisión varía en profundidad poco después de la unión en vértice de los dos trazos internos volviéndose más finos y superficiales. El trazo externo izquierdo se bifurca en el tercio inferior, prolongándose la rama interna hasta alcanzar la línea de fractura. La altura del signo es considerablemente mayor que la del primero: unos $25 \mathrm{~mm}$. mide aproximadamente el trazo exterior derecho, mucho más limpio que el izquierdo, que llega hasta los $31 \mathrm{~mm}$.

En apoyo de una transcripción ś frente a ko, además de las peculiaridades en el trazado ya señaladas, cabe apuntar la ausencia de la variante ko3 en Azaila (siempre ko se presenta bajo la variante ko1) y, al margen de argumentos paleográficos, comprobaremos inmediatamente que con ś obtenemos una secuencia que se acomoda bien en el léxico ibérico

El tercer signo, ke1, de $25 \mathrm{~mm}$ de altura, no presenta peculiaridades, si acaso habría que señalar una apertura del ángulo que forman sus dos trazos superior a la habitual. El extremo del trazo superior está afectado ya por la erosión que ha acabado con el barniz y arrastra las primeras capas de arcilla, habiendo borrado prácticamente por completo el último signo.

De lo que fue el último grafema sólo se conserva el inicio de dos trazos que se unen en vértice. A partir de ellos pueden reconstruirse varios signos (be, te, $\mathbf{k u}, \mathbf{r}, \mathbf{r}$...), hemos optado por una transcripción '́r (o también r) porque con ella componemos el texto más familiar al léxico ibérico: (i)śkeŕ, (e)śkeŕ, conocido componente antroponímico ${ }^{7}$ que puede presentarse con cualquiera de las dos sibilantes y con cualquiera de las dos vibrantes.

Puesto que no existe testimonio alguno de un componente onomástico tiśkeŕ, debemos pensar en la unión de un primer elemento terminado en ti o te con un segundo (i)śkeŕ /(e)śkeŕ, por medio de la contracción de dos vocales o la elisión de la vocal inicial del segundo elemento. Podríamos así recomponer, basándonos en el repertorio conocido hasta ahora, un hipotético ulti(e)śkeŕ / ulti(i)śkeŕ e incluso ete(i)śkeŕ que vendría a engrosar el capítulo de NNP claramente ibéricos identificados en Azaila ${ }^{8}$. Entre éstos recordemos que uno contiene el componente eskeŕ: aiuneske ŕ, sobre ánfora romana, y otro el componente ete: eteśike, en una pátera campaniense 5/7Lamb. n. 22 .

Bibliografía: Cabré 1944, fig. 19 n. 240; Beltrán 1979, p. 163, fig 9

\footnotetext{
${ }^{7} \mathrm{~N}^{\mathrm{o}} 64$ del repertorio de J. Untermann (MLH III, 1, pp. 224-225).

${ }^{8}$ Hay un grupo de NNP no identificables que, según De Hoz (1993, p. 654), pueden pertenecer a un estrato lingüístico no ibérico que puede ser o no el mismo que aisla en Ullastret.
} 
E. 1.291: etaute[/¿̇ube?

Nueva lectura: etaute[ / $\underline{\text { etaute }}$

MAN 1943/69/607

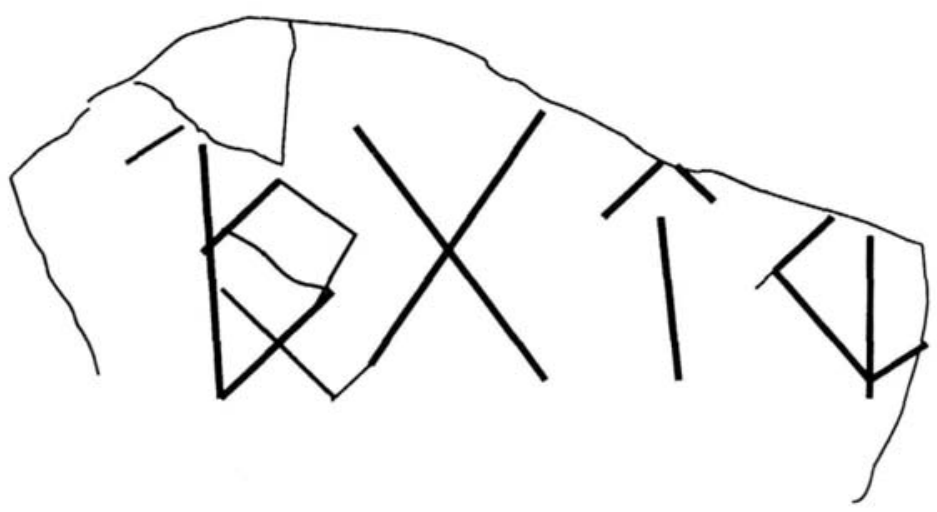

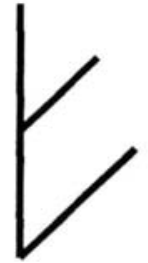

$1^{\circ}$

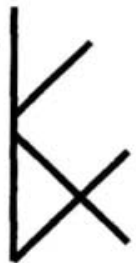

$2^{\circ}$

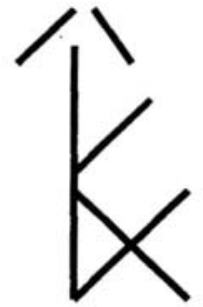

$3^{\circ}$

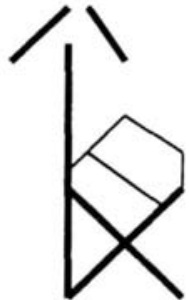

$4^{\circ}$

Figura 2. En la parte superior calco del epígrafe E. 1.291, según J. Valladolid. En la parte inferior desarrollo del monograma del primer signo.

Fragmento de pared de una tinaja indígena de barro rojizo sin pintar, de $9,6 \times 8,2 \mathrm{~cm}$.

La inscripción, grabada postcocción, consta de 4 grafemas, los tres primeros completos y el cuarto interrumpido por la línea de fractura, pero fácilmente restituible a partir de los trazos existentes: e1, ta1, u1, te4. Puede estar incompleta por la derecha. Los trazos son limpios, anchos y profundos. La altura media de los signos es de $28 \mathrm{~mm}$ y forman una línea de $75 \mathrm{~mm}$ de longitud.

La peculiaridad de la inscripción gira en torno al primer signo. En el campo epigráfico que ocupa se distinguen además de los trazos que dan forma al grafema e, otros que admiten ser transcritos finalmente como un nexo de varios signos, que en $M L H$ se lee ¿ube? En efecto el trazo vertical de e ha sido utilizado como parte de la letra $\mathbf{u}$, añadiendo los 
trazos oblicuos cortos en su extremo superior para completarla, de los cuales sólo podemos ver hoy el de la izquierda, ya que el derecho se ha perdido por rotura de esa parte del fragmento cerámico. Tenemos por tanto la letra u contenida en el mismo campo y en claro nexo con la letra e. El siguiente grafema que identificamos es ta para el que también se ha utilizado uno de los trazos de e, el oblicuo inferior, con el que formaría nexo. Otro signo que se aisla -aunque no tan nítidamente- es te que se ha servido del trazo oblicuo superior de e, de la mitad superior de ta y de una zona del vástago vertical que participa en la formación de e y $\mathbf{u}$, de manera que queda ligado con todos ellos (fig. 2). Si situamos todos estos signos en línea, nos encontramos con la serie etaute, la misma que nos ofrecen los 4 signos individualizados que componen la inscripción. Podríamos concluir que la primera letra puede estar encerrando, en monograma, la misma palabra que se lee en el epígrafe completo. La transcripción que ofrecemos por tanto sería: etaute/etaute.

Hay un aspecto que juega en contra de esta interpretación: las distintas variantes que presenta el grafema te: te 2 en el monograma y te4 en la secuencia en línea. Esta incoherencia gráfica podría resolverse con la lectura '́ del último signo, que daría al traste con la duplicidad de textos planteada pero no modificaría la transcripción del monograma. Puesto que tanto etaute como etauŕ pueden admitirse en el léxico ibérico, acudimos a la paleografía para conocer cuál de las dos variante gráficas te4 o ŕ4 tiene mayor presencia en Azaila, comprobando que ninguna de ellas cuenta con representación en el conjunto epigráfico de este yacimiento. Optamos finalmente por la lectura etaute empleando el único indicio de la coincidencia entre el monograma y los 3 primeros signos de la inscripción en línea.

No está testimoniada otra secuencia etaute en el léxico ibérico, sí en cambio es frecuente, precisamente en Azaila, la más corta taute que aparece siempre con sus tres signos ligados (E. 1.219-221 y 340-341) (Figura 4). También es habitual en forma más abreviada: tau. El componente onomástico de mayor semejanza léxica sería tautin, sin presencia en Azaila, al menos en su forma completa, pero conocido en el vecino yacimiento del Castelillo de Alloza, también en Teruel. Aún llamando la atención la proximidad léxica entre etaute y taute, así como la coincidencia en la "sofisticación" de la presentación (taute en nexo trilítero y etaute en monograma), no podemos relacionarlas con un mismo individuo (propietario, comerciante...) puesto que no hay constancia de que se hallasen en la misma casa.

Bibliografía: Cabré 1944, p. 32 n.50, fig. 18 n. 50.

\footnotetext{
${ }^{9}$ Son relativamente frecuentes en Azaila ligaduras entre dos o más signos, algunas de gran complejidad como éstas. La paleografía en Azaila lejos de carecer de interés, como podría pensarse por hallarnos ya ante un signario totalmente evolucionado e inmerso en un periodo cronológico corto (prácticamente todos los epígrafes pueden fecharse entre fines del II y primer cuarto del I a.C.), participa del entramado de enigmas que encierra el conjunto epigráfico de Azaila, cuya resolución será el único camino para entender un yacimiento ya prácticamente agotado desde el punto de vista estrictamente arqueológico y sin embargo todavía no plenamente "descifrado".
} 
E. 1.78: bas o sba.

Nueva lectura bas/bas o sba/sba

MAN 1943/69/378

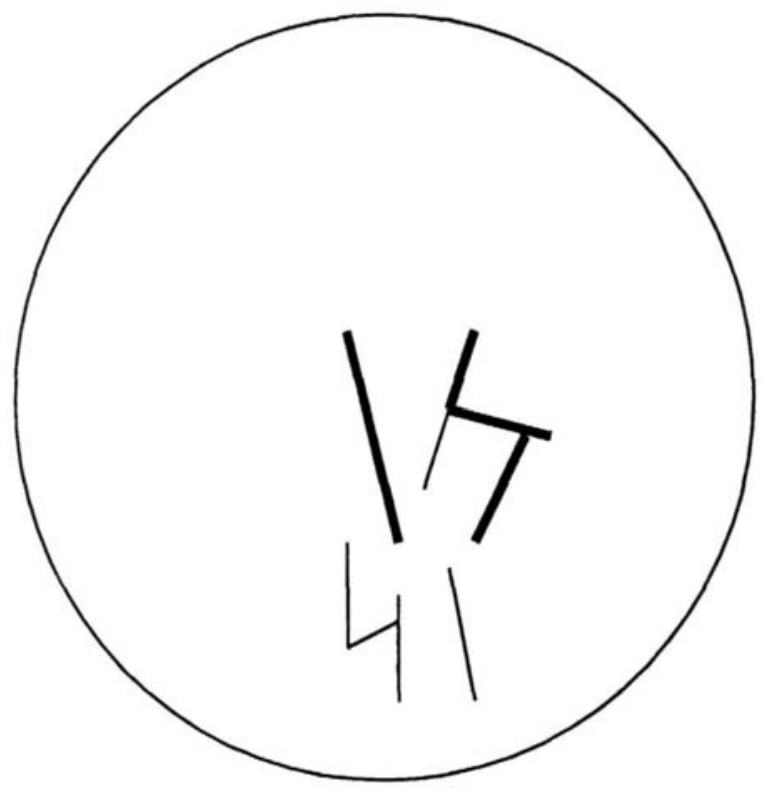

Figura 3. E. 1.78

Base completa y pared sin borde de cerámica campaniense $\mathrm{B}$, forma 5/7 Lamb. Pasta beige amarillenta depurada y compacta. Barniz grisáceo. Fondo exterior sin barnizar. Diámetro de la base: 9,9 cm. bas/bas.

En el fondo exterior presenta dos grafitos de idéntica transcripción:

El más visible por su posición casi central y su mayor módulo, lo componen dos signos ba1 y s1 de $19 \mathrm{~mm}$ de altura, que forman una línea de $15 \mathrm{~mm}$ de longitud. El trazo es grueso y profundo, con prolongaciones de los trazos de $\mathbf{s}$ más allá de las uniones entre ellos.

El segundo, más próximo al arranque del pie, consta también de dos grafemas: ba1 y s1, de trazos finos, de menor profundidad y con módulo más pequeño que el del grupo anterior. Todo ello ha hecho que haya pasado desapercibido. En las dos publicaciones donde se recoge esta pieza, sólo se dibuja el grupo primero y junto a él (debajo o sobre, en función de la posición de lectura que adoptemos) dos trazos verticales, que no son otra cosa que parte de este segundo grafito.

Dadas las diferencias de trazado entre ambos grupos, creemos que se trata de dos textos independientes y desechamos por tanto una lectura única basbas.

\footnotetext{
${ }^{10}$ Cabré (1944), fig. 21 n. 266 y MLH E. 1.78.
} 
Revisión de seis inscripciones sobre cerámica procedentes de Azaila (Teruel)

Es imposible conocer los motivos que llevaron a los habitantes de Azaila a grabar en una misma pieza dos grafitos iguales, fenómeno habitual en este yacimiento ${ }^{11}$, pero en el caso que nos ocupa, tal vez la primera grabación no resultase suficientemente vistosa y se repitiese con trazos más grandes y en el centro de la base, haciéndola así perfectamente visible; lo que vendría a sancionar la importancia que en el oppidum de Azaila se otorgaba a la distinción de los vasos cerámicos (fundamentalmente a los de importación) por medio de las marcas gráficas.

En bas reconocemos un elemento onomástico ibérico ampliamente testimoniado

Bibliografía: Cabré 1944, fig. 21 n. 266.

MLH: E. 1.219. si/tau

Nueva lectura: tautabas

MAN: $1943 / 69 / 368$

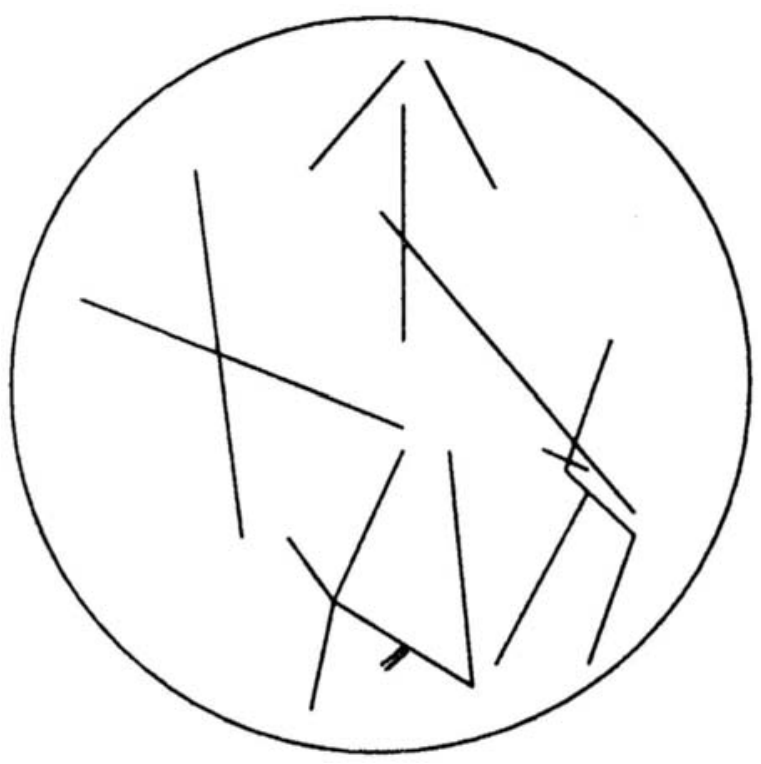

Figura 4. E. 1.219

Base completa y pared sin borde de cerámica campaniense B, forma 5/7 Lamb. La pasta es ocre-amarronada y el barniz está muy deteriorado tanto en el exterior como en el interior. La base tiene un diámetro de $7 \mathrm{~cm}$ El fondo interior está decorado con círculos concéntricos y ruedecilla

\footnotetext{
${ }^{11}$ Acabamos de ver la repetición de etaute. Otros ejemplos: bateba/bateba (E.1.85), la/la (E. 1.165), kuY/kuY (E. 1.158), entre otros.

${ }^{12} \mathrm{~N}^{\circ} 27$ del repertorio de Untermann (MLH III, 1, pp 214-215).
} 


\section{Juana Valladolid}

El fondo exterior está totalmente ocupado por una inscripción grabada postcocción.

En los $M L H$ se informan dos textos diferenciados: tau/si, que en efecto pueden aislarse del conjunto de trazos más o menos intencionados que salpican toda la base del plato. Nosotros hemos optado por una transcripción tautabas, obtenida también a partir de esa misma maraña.

La primera parte de la inscripción no tiene dificultad de lectura (ta1 u1), sólo un arañazo que recorre toda la base afecta al segundo signo. La altura de los signos es de 14 y $12 \mathrm{~mm}$, respectivamente. Sin modificar la posición de lectura puede identificarse la forma de ta, aunque distorsionada, ba y s. Entre ba y s un rasguño, más que trazo voluntario, parece unir ambos signos, mientras que s está también envuelta en trazos que ensucian su dibujo. De una de las aspas de lo que nosotros consideramos ta parten dos pequeños trazos que son los que autorizarían una transcripción i, siempre que cambiemos la posición de lectura girando la base $180^{\circ}$.

Ya hemos señalado la frecuencia de la secuencia tau en Azaila, tal vez abreviatura de NP, en esta ocasión si la unimos a tabas, nos recuerda, por ejemplo, al Teitabas de la Tabula Contrebiensis, aunque con distintos digptongos.

Bibliografía: Cabré 1944, p. 35 n. 269, fig 21 n. 269; Beltrán 1979, p. 188 y fig. 20 n. 194.

\section{E. 1.294 kite[}

Nueva lectura: bate[ba]

MAN 1943/69/2635

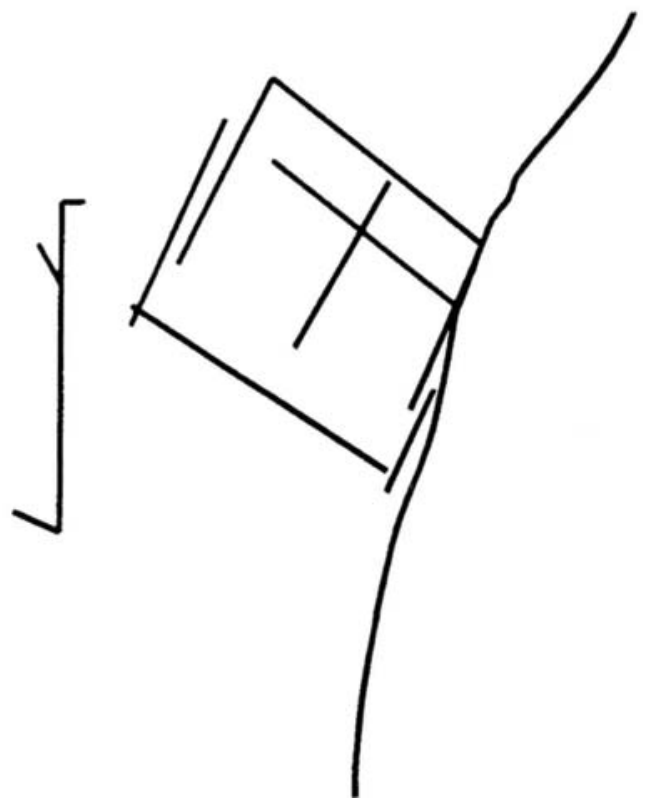

Figura 5. E. 1.294 
Revisión de seis inscripciones sobre cerámica procedentes de Azaila (Teruel)

Fragmento de base y pared de un plato indígena sin pintar, imitación de una forma $5 / 7$ Lamb. de cerámica campaniense B. Las paredes son finas y la pasta rojiza. La base tiene un diámetro de $8,1 \mathrm{~cm}$.

La inscripción se grabó postcocción en el exterior de la pared y consta de dos grafemas completos: ba1, te6, de trazos finos y profundos. La altura de los signos es de $20 \mathrm{~mm}$ y hasta la línea de fractura forman una línea de $20 \mathrm{~mm}$ de longitud.

El primer signo además de una mínima bifurcación en su tercio superior presenta dos marcas de aproximadamente $2-3 \mathrm{~mm}$ de longitud en cada uno de sus extremos que pueden legitimar una transcripción ki; sin embargo, siempre que el grafema ki aparece en Azaila lo hace con sus trazos cortos mucho más desarrollados y generalmente bajo la variante ki2, con un trazo principal marcadamente oblicuo. El segundo signo se presenta bajo la variante te 6 que en Azaila sólo la vemos cuando forma parte de la serie gráfica bateba ${ }^{13}$, en el resto de epígrafes las formas habitualmente empleadas fueron te 2 y te1. Aún siendo éste un argumento paleográfico valioso, la defensa de una lectura ba descansa fundamentalmente en la frecuencia en Azaila de la secuencia bateba que Cabré ${ }^{14}$ identificó sobre campaniense B, ánfora vinaria y "en el dorso de una imitación indígena sin negro brillante de un C I" (Lamb.5/7), definición que se aviene con la pieza que estamos estudiando.

Por otra parte, en el álbum de dibujos de Cabré publicados en 1995 por Beltrán ${ }^{15}$ se recoge un fragmento cerámico de estas características y con la secuencia bateba inscrita, cuyo primer signo se dibuja con la misma bifurcación que tiene el que estamos analizando, aunque Cabré no reflejaba las dos pequeñas incisiones que remarcaban los extremos del trazo principal. Asimismo el segundo signo presenta un dibujo prácticamente superponible, por lo que creemos podría tratarse de la misma pieza, aunque en la actualidad el fragmento que se conserva es más pequeño que el que vio Cabré y sólo contiene ya los dos primeros signos.

En bateba se ha visto un NP perteneciente probablemente a un estrato lingüístico no ibérico ${ }^{16}$

Bibliografía: Cabré 1944, p. 33 n. 161; Beltrán 1995, fig 32, n. 2.

E. $1.240: * * * \mathbf{l k a}$

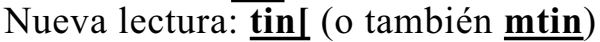

MAN: $1945 / 69 / 261$

Fragmento de base de cerámica campaniense B, forma 3 Lamb. La pasta es ocre y el barniz negro, denso y con irisaciones. Tiene un diámetro de $11,5 \mathrm{~cm}$.

\footnotetext{
${ }^{13} \mathrm{E} 1.84-88,317,369$

${ }^{14}$ Cabré (1944) p. 33 n. 161

${ }^{15}$ Beltrán Lloris (1995), fig. 32 n. 2.

${ }^{16}$ De Hoz (1993), cit. nota 8
} 


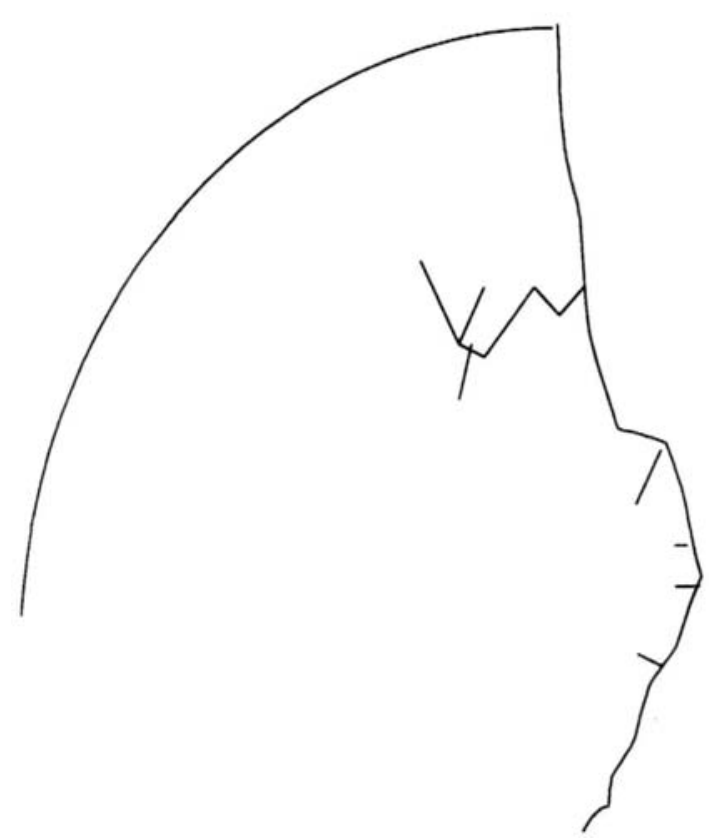

Figura 6. E. 1.240

En el fondo exterior se grabaron postcocción dos grafemas completos ligados (iㅡ) de $12 \mathrm{~mm}$ de altura y $15 \mathrm{~mm}$ de longitud. Los trazos son finos y poco profundos. En torno al centro de la base hay repartidos varios trazos cortos interrumpidos por la línea de fractura que debieron formar parte de esta misma o quizá de otra inscripción.

Lo más significativo del epígrafe es la forma que presenta el signo ti, distinta a las reconocidas en las tablas de formas de $M L H$. La variante más próxima es ti2 y la diferencia con ella estriba en que el brazo central del tridente desaparece en favor de un trazo oblicuo que se une en vértice con el brazo izquierdo. El aspecto final permite también una transcripción como nexo entre $\mathbf{Y}$ y ti. Esta peculiar forma de ti o de nexo Yti se repite en Azaila en otros epígrafes, fundamentalmente sobre pesas de telar y con distintas variantes (figura 9), a veces la forma de partida no es ti2 sino ti4 (vid. E 1.360).

La transcripción lka $(M L H)$ parte de una posición de lectura contraria (desde el interior hacia el exterior) y sobre todo de un calco en el que no se considera el trazo vertical inferior del signo que nosotros leemos ti.

La secuencia tin, también en ligadura, se repite en E. 1.66

Ante la imposibilidad de saber si estamos ante una agrupación independiente o si formaba parte de una inscripción más extensa, cualquier aproximación interpretativa, más allá de la consideración como marca de propiedad, sería improcedente. 


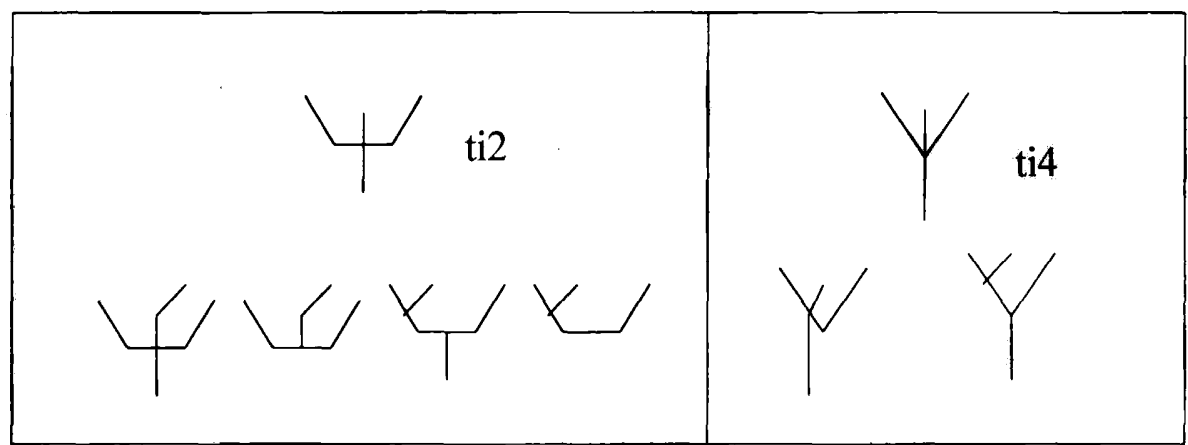

a

b

Figura 7. Variantes anómalas de la grafía ibérica ti identificadas en Azaila (¿nexos?):

a) a partir de ti2, b) a partir de ti4

3. Hasta ahora las nuevas lecturas obtenidas, tanto las aquí presentadas como las que no hemos considerado de interés comentar pero que encontrarán su lugar en el Banco de Datos Lingüísticos Paleohispánicos, pertenecen a epígrafes revisados directamente en el MAN, donde se conservan la mayor parte de materiales del yacimiento del Cabezo de Alcalá de Azaila (Teruel). Por lo tanto todavía puede haber inscripciones, custodiadas en otros museos, susceptibles de ofrecer alguna novedad y que estamos pendientes de revisar.

\section{BIBLIOGRAFÍA}

BELTRÁN LLORIS, M. (1976): Arqueología e Historia de las ciudades antiguas del Cabezo de Alcalá de Azaila (Teruel), Monografías Arqueológicas 19, Zaragoza.

(1979): "La cerámica campaniense de Azaila. Problemas de cronología del valle medio del Ebro", Caesaraugusta 47-48, pp. 141-209.

(1995): Azaila. Nuevas aportaciones deducidas de la documentación inédita de J. Cabré Aguiló, Institución "Fernando el Católico". Zaragoza.

CABRÉ AGUILÓ, J. (1926): "La cerámica pintada de Azaila", Archivo Español de Arte y Arqueología VI, pp. 215-160.

(1944): Cerámica de Azaila. Museos Arqueológicos de Madrid, Barcelona y Zaragoza. CVH., Madrid. 


\section{Juana Valladolid}

DE Hoz, J. (1993): "La lengua y la escritura ibéricas". Lengua y Cultura en la Hispania prerromana. Actas del V Coloquio sobre lenguas y culturas prerromanas de la Península Ibérica (Colonia 25-28 de noviembre de 1989), eds. J. Untermann y F. Villar, pp. 635-66, Salamanca.

UNTERMANN J. (1990): Monumenta Linguarum Hispanicarum Bd III. Die Iberischen Inschriften aus Spanien, Wiesbaden.

Juana Valladolid Moya

Universidad Autónoma de Madrid e-mail:jvalladolid33@hotmail.com 
Revisión de seis inscripciones sobre cerámica procedentes de Azaila (Teruel)

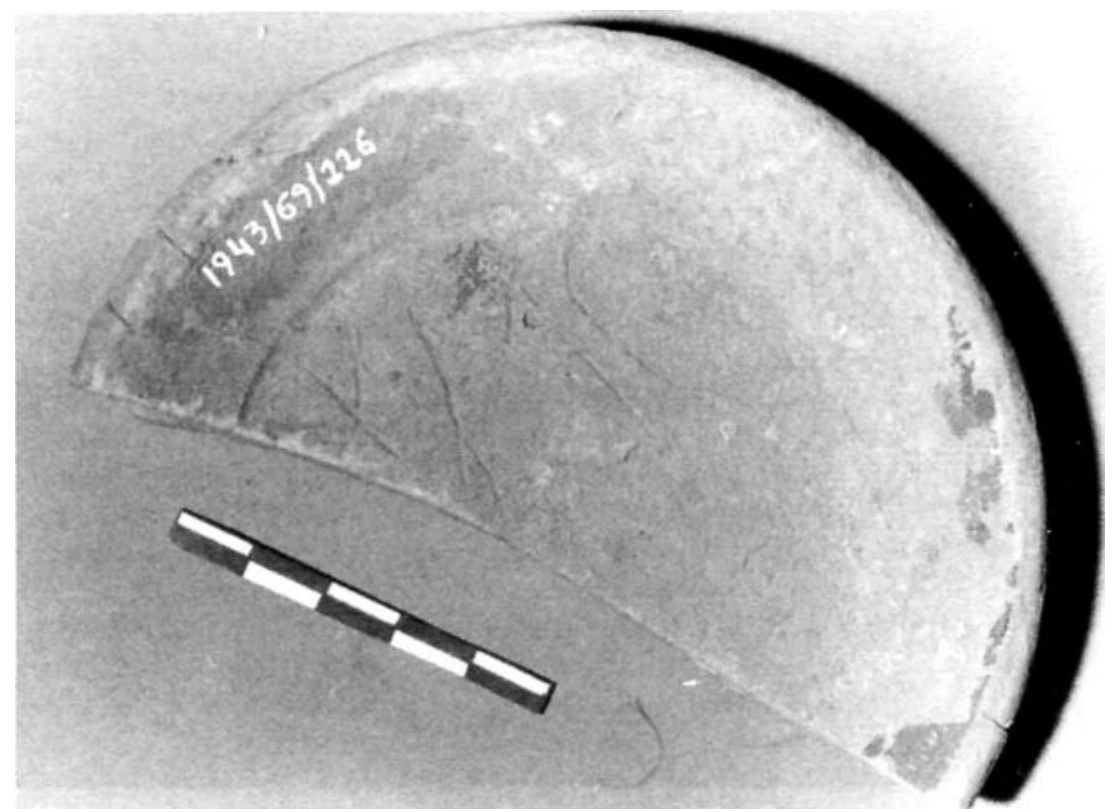

Fig. 8. E. 1.223 (fotografía: J. Valladolid).

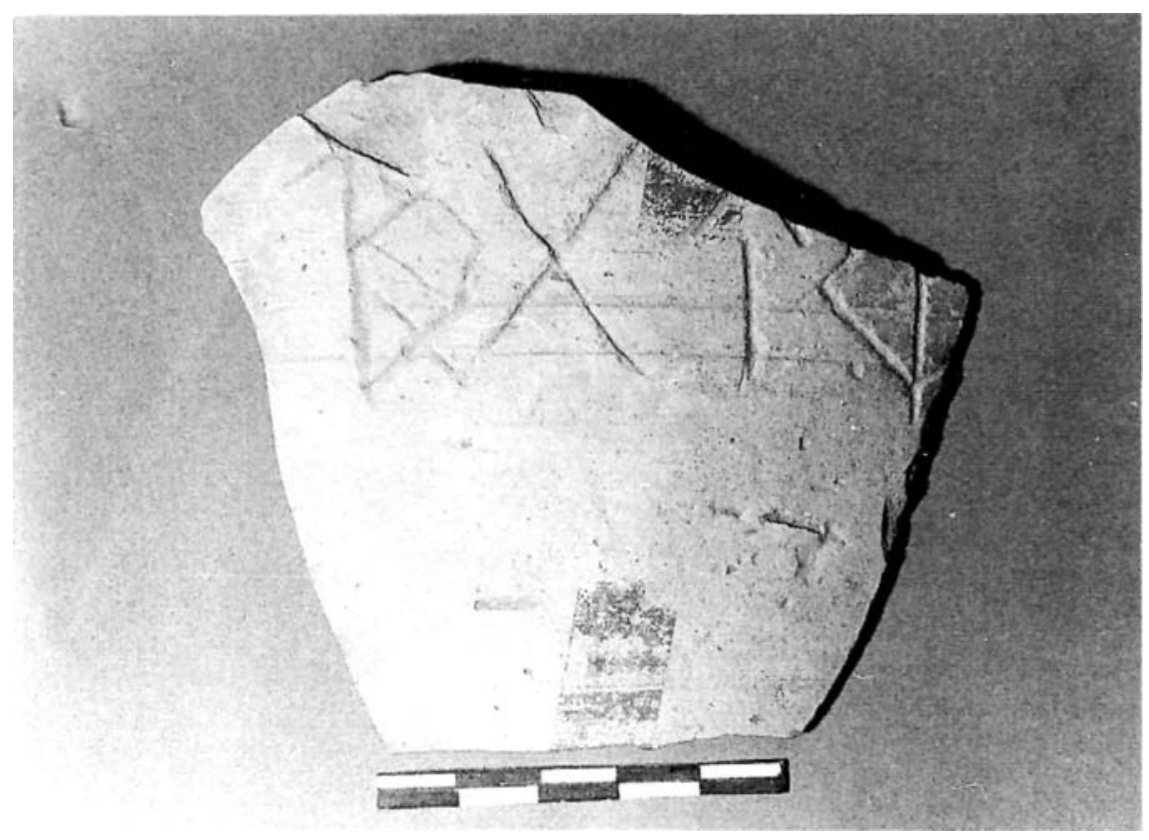

Fig. 9. E. 1.291 (fotografía: J. Valladolid). 


\section{Juana Valladolid}

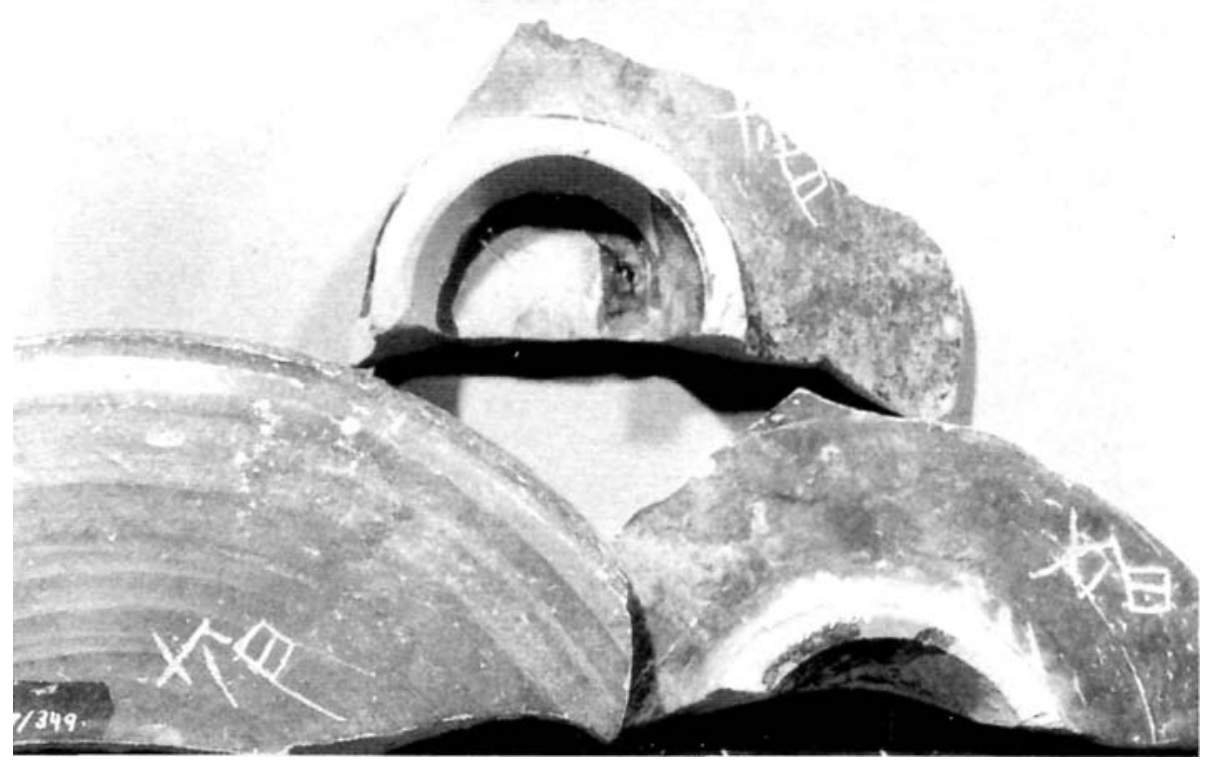

Fig. 10. La secuencia taute en materiales de Azaila (fotografía: J. Valladolid).

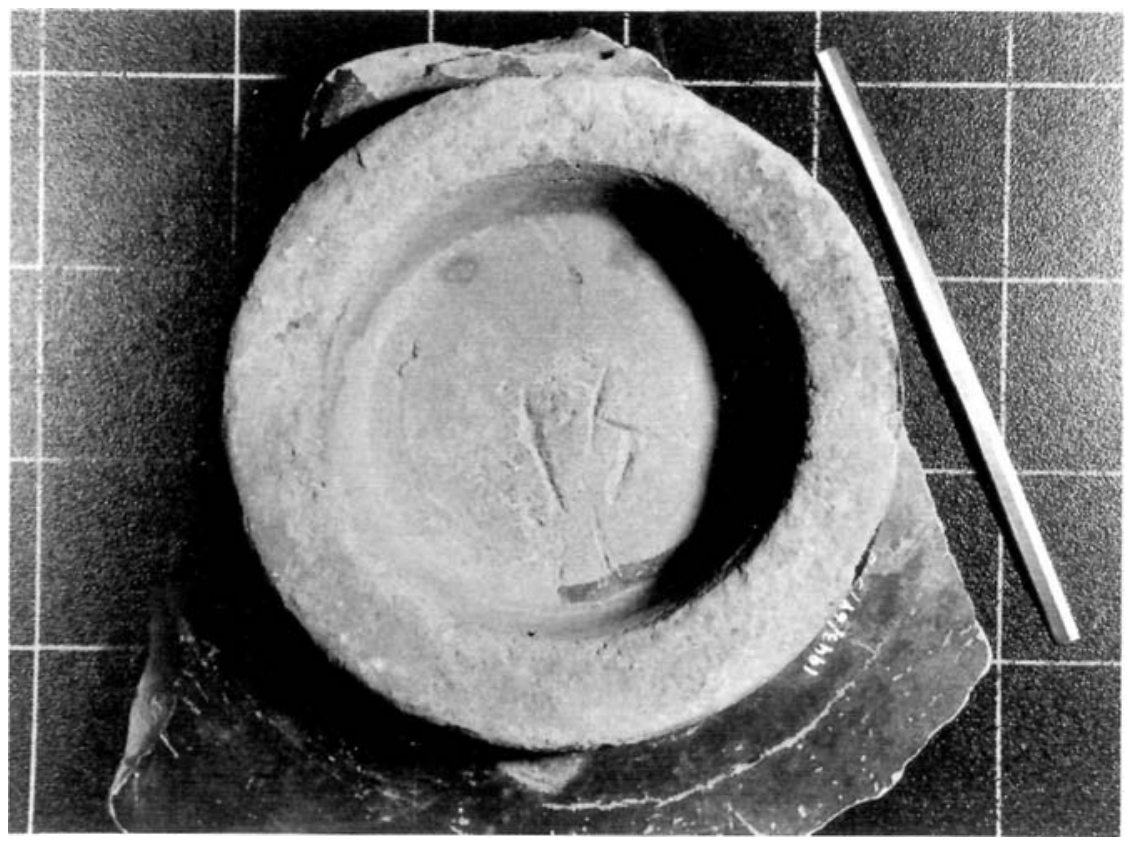

Fig. 11. E. 1.78 (fotografía: J. Valladolid). 
Revisión de seis inscripciones sobre cerámica procedentes de Azaila (Teruel)

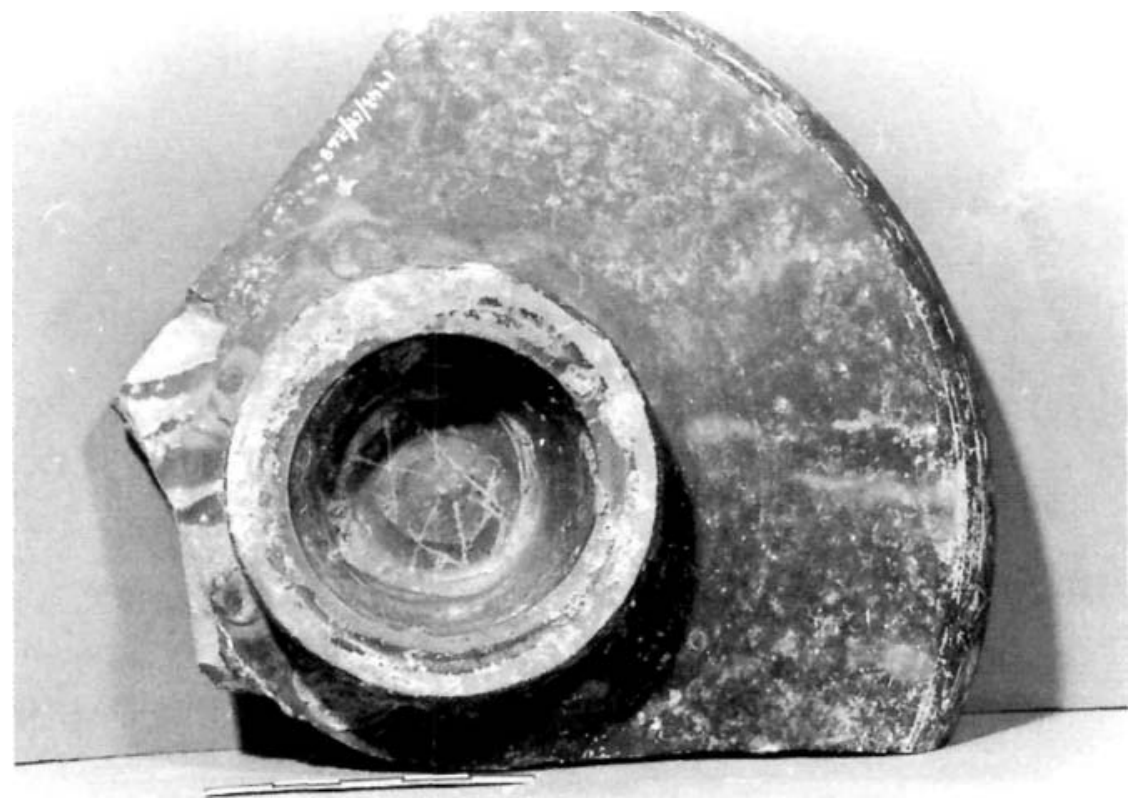

Fig. 12. E. 1.219 (fotografía: J. Valladolid).

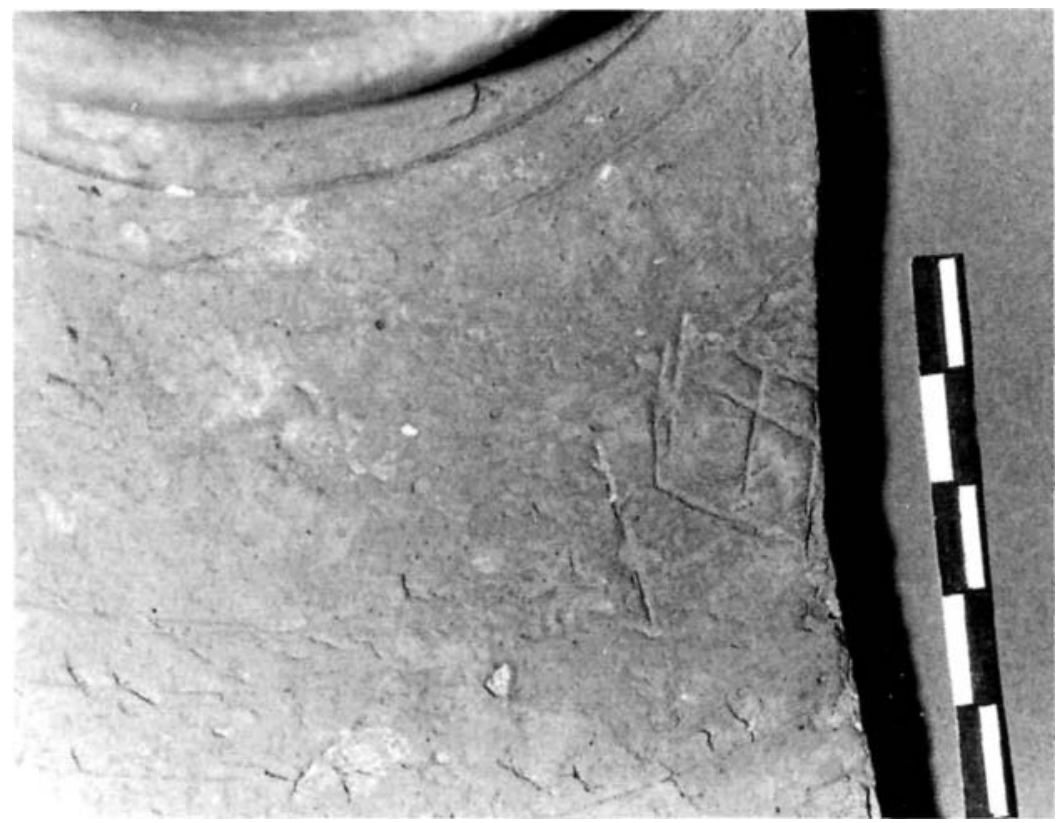

Fig. 13. E. 1.294 (fotografía: J. Valladolid). 


\section{Juana Valladolid}

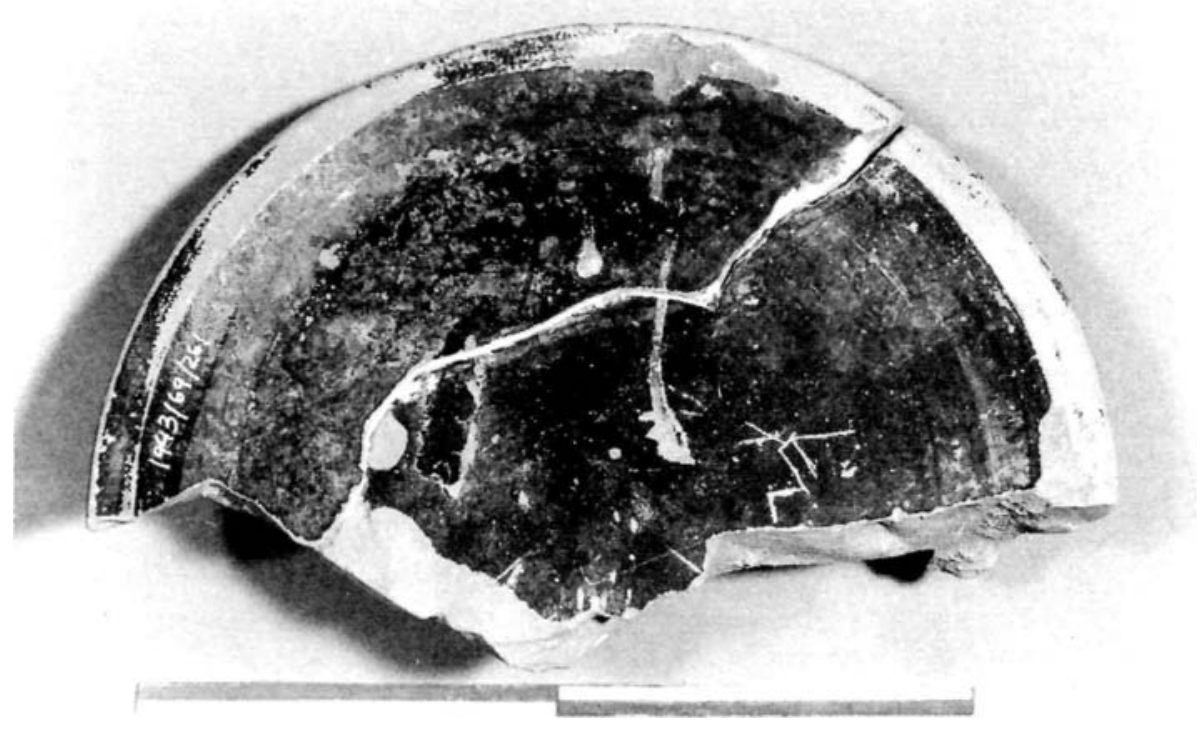

Fig. 14. E.1.240 (fotografía: J.Valladolid). 